\title{
The submersion of sodium clusters in helium nanodroplets: Identification of the surface $\rightarrow$ interior transition
}

\author{
Lukas An der Lan, ${ }^{1}$ Peter Bartl, ${ }^{1}$ Christian Leidlmair, ${ }^{1}$ Harald Schöbel, ${ }^{1}$ Roland Jochum, ${ }^{1}$ \\ Stephan Denifl, ${ }^{1}$ Tilmann D. Märk, ${ }^{1}$ Andrew M. Ellis, ${ }^{2, \text { a) }}$ and Paul Scheier ${ }^{1, \text { a) }}$ \\ ${ }^{1}$ Institut für Ionenphysik und Angewandte Physik und Research Platform Advanced Materials, \\ Universität Innsbruck, Technikerstr. 25, A-6020 Innsbruck, Austria \\ ${ }^{2}$ Department of Chemistry, University of Leicester, University Road, Leicester LE1 7RH, United Kingdom
}

(Received 15 April 2011; accepted 23 June 2011; published online 27 July 2011)

\begin{abstract}
The submersion of sodium clusters beyond a critical size in helium nanodroplets, which has recently been predicted on theoretical grounds, is demonstrated for the first time. Confirmation of a clear transition from a surface location, which occurs for alkali atoms and small clusters, to full immersion for larger clusters, is provided by identifying the threshold electron energy required to initiate $\mathrm{Na}_{n}$ cluster ionization. On the basis of these measurements, a lower limit for the cluster size required for submersion, $n \geq 21$, has been determined. This finding is consistent with the recent theoretical prediction. @ 2011 American Institute of Physics. [doi:10.1063/1.3610388]
\end{abstract}

\section{INTRODUCTION}

Alkali atoms are notoriously reluctant to enter helium nanodroplets. The preference for a surface location stems from the relatively diffuse $s$ valence electron in each alkali atom, which produces a strong Pauli repulsion that overwhelms the weak dispersion force between the alkali and helium atoms. As demonstrated by spectroscopic techniques and confirmed by theoretical studies, the alkali atom sits in a dimple on the helium droplet surface. ${ }^{1-3}$

Pauli repulsion is equally dominant for small alkali clusters, keeping them on the surface of the liquid helium. However, some interesting and subtle effects also come into play. For small clusters, the formation of spin paired metal-metal bonds releases a large amount of energy, leading to expulsion of the cluster into the gas phase as this energy is dissipated. On the other hand, high spin clusters can survive on the surface of the helium droplet because of the much weaker metal-metal interactions derived from parallel spin configurations. Consequently, at least for small alkali clusters, we have the remarkable situation where cluster formation on helium nanodroplets leads to the selective survival of high spin clusters. ${ }^{4-6}$ For larger clusters it has been suggested that the formation of spin-polarized configurations is of low probability, so most likely these clusters will be in their electronic ground states. ${ }^{7}$ Important evidence in support of this suggestion has been derived recently from detailed modelling of alkali cluster distributions. ${ }^{8}$ The model takes into account factors that can lead to a deviation from the more commonly used Poissonian statistics used to model cluster distributions in helium droplets, i.e., droplet shrinkage as each atom is added, cluster desorption due to energy release, and momentum transfer during the dopant-droplet collision. A comparison of experimental alkali cluster size distributions, derived from photoionization mass spectrome-

\footnotetext{
a) Authors to whom correspondence should be addressed. Electronic addresses: Andrew.Ellis@le.ac.uk and Paul.Scheier@uibk.ac.at.
}

try of alkali-doped helium nanodroplets, with this new model suggests that selective production and retention of high spin clusters is only important for relatively small cluster sizes.

Although small alkali clusters are known to reside on the surface of helium nanodroplets, there is the possibility that larger clusters could sink inside. The driving force for such a change would be a favourable dispersive attraction between the clusters and the surrounding helium, which would need to offset both the Pauli repulsion and the surface energy expended in creating a sufficiently large bubble inside the helium droplet (within which to locate the cluster). With any increase in size of the cluster the dispersion force grows, essentially through an increase in the number of atom-atom interactions. An alternative way of looking at this is to recognise that the polarizability of alkali clusters increases with size. Thus, at some critical size the cluster may become submerged in the helium rather than adopt a surface location. Recently, Stark and Kresin have described and employed a theoretical model which anticipates such a critical cluster size for alkali submersion. ${ }^{9}$ For example, $\mathrm{Na}_{n}$ clusters are predicted to preferentially enter ${ }^{4} \mathrm{He}$ nanodroplets once $n \geq 21$, whereas $\mathrm{K}_{n}$ clusters require approximately 78 atoms to become fully solvated.

So far no experiment has identified this surface $\rightarrow$ interior transition. Here, we provide the first such experimental demonstration, using sodium clusters as the target species. The method employed builds upon previous work by Vongehr et al. in which electron-induced ionization was applied to $\mathrm{Na}_{n}$ clusters on helium nanodroplets. ${ }^{10}$ By exploring the electron energy dependence of the ionization process, and in particular identifying cluster ions produced by Penning ionization, Vongehr et al. presented evidence showing that clusters containing up to at least $13 \mathrm{Na}$ atoms reside on the helium droplet surface. Here, we extend these studies to much larger clusters sizes and are able to see a distinct change in ionization behavior which provides the first evidence for a clear onset of alkali cluster submersion in helium nanodroplets at a specific cluster size. 


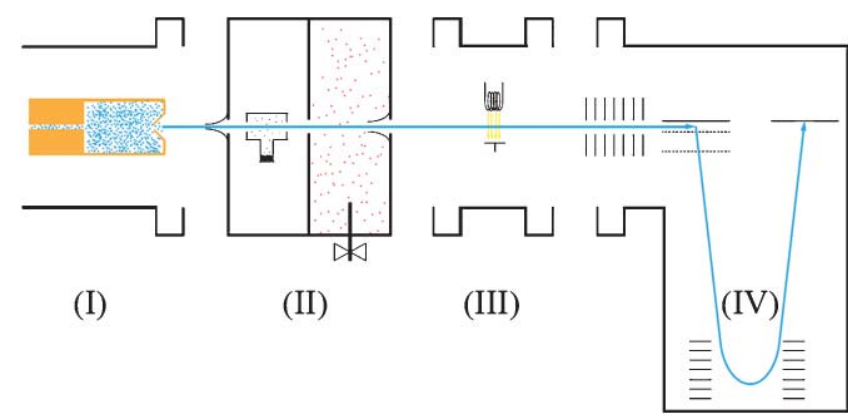

FIG. 1. Schematic of the experimental apparatus. The helium nanodroplets are generated by supersonic expansion in the cluster source region (I) and pass through a $10 \mathrm{~cm}$ to $20 \mathrm{~cm}$ long pick-up region (II) before they enter the ionization chamber (III). In this chamber the nanodroplets interact with an electron beam and cations are formed. The resulting cations are then mass analyzed by a time-of-flight mass spectrometer (IV).

\section{EXPERIMENTAL}

This investigation was performed using a new experimental setup in Innsbruck. An overview of the apparatus is first given here, before we consider aspects of the equipment and procedures employed in more detail. An illustration of the apparatus is provided in Figure 1. A beam of neutral helium nanodroplets is formed in the cluster source (I), which then enters a pick-up region (II), where the droplets can be doped with atoms or molecules. After leaving the pick-up zone the helium nanodroplets interact with an electron beam in the ionization region (III). Finally, the resulting cations are mass-analyzed by an orthogonal reflectron time-of-flight mass spectrometer and detected by a microchannel plate assembly (IV).

For the experiments reported in this paper, neutral helium droplets were formed by supersonic expansion of ${ }^{4} \mathrm{He}$ (99.9999\% purity) through a $5 \mu \mathrm{m}$ aperture in a platinum disk. The source is mounted on a translation stage for easy alignment and is connected by copper braids to the second stage of a closed-cycle refrigerator (SRDK-415D-F50H, Sumitomo Heavy Industries Ltd.). The braids serve as a thermal connection between the nozzle and the refrigerator unit while also enabling the source to be decoupled from the vibrations of the cryocooler. The ultimate temperature achievable with this arrangement was found to be $5.5 \mathrm{~K}$ under helium gas load (stagnation pressure of $20 \mathrm{bar}$ ). The temperature is measured by a silicon diode and controlled by a resistive heater. Both devices are operated by a Lakeshore temperature controller (Model 331 Cryogenic Temperature Controller). The stagnation pressure can be varied between 15 and 30 bars and the temperature of the nozzle is typically operated between $5-15 \mathrm{~K}$. Within this range of parameters we can achieve both subcritical and supercritical expansion conditions, where the average helium droplet size $\langle N\rangle$ changes from $\sim 10^{4}$ to $10^{5}-$ $10^{6}$ helium atoms. ${ }^{11}$ The pressure in the expansion chamber is kept below $10^{-4}$ mbar by two turbomolecular pumps with a total pumping speed of $3800 \mathrm{l} / \mathrm{s}$ for helium. For the specific work performed in the current study a nozzle temperature of $5.7 \mathrm{~K}$ and a stagnation pressure of 25 bar was used, giving $\langle N\rangle$ $\approx 10^{5}$ helium atoms.
After formation, the He droplet beam passes through a skimmer with a $0.8 \mathrm{~mm}$ aperture and enters a differentially pumped chamber, where the pick-up processes take place. The length of a single pick-up chamber is $\sim 10 \mathrm{~cm}$ and can by extended to $20 \mathrm{~cm}$ by adding a second pick-up chamber, if required. Depending on the goal of the study, each pick-up chamber can be sub-divided into two differentially pumped regions for multiple pick-up processes or equipped with one or two heating cells. The base pressure in the pick-up chamber was kept below $10^{-6}$ mbar by two 70 1/s turbomolecular pumps. For the experiments reported here, a pick-up cell with a length of $6 \mathrm{~cm}$ was used. Sodium vapour was generated by oven evaporation and both the oven and the pick-up cell were heated to $270^{\circ} \mathrm{C}$, which should correspond to a sodium vapour pressure of approximately 5 $\times 10^{-3}$ mbar. Due to the additional pumping in the pick-up region the local sodium pressure encountered by the helium droplet beam is expected to be significantly lower but was not measured.

Leaving the pick-up region, the nanodroplet beam enters the differentially pumped ionization chamber through a skimmer with a $2 \mathrm{~mm}$ diameter aperture. The base pressure in the ionization region is below $10^{-7}$ mbar. In this chamber, an electron beam of variable energy (from 0 to $150 \mathrm{eV}$ ) and current (from 10 to $200 \mu \mathrm{A}$ ) crosses the helium droplet beam. The electrons are emitted from a tungsten-rhenium filament and guided by a low magnetic field to maximise overlap with the helium droplet beam. Depending on the electron energy, electron impact ionization or electron attachment studies can be carried out. In the current work, the electron energy was varied between 16 and $30 \mathrm{eV}$ in $1 \mathrm{eV}$ steps in order to create positive ions. The electron energy resolution was approximately $1 \mathrm{eV}$. The ions are guided by a weak electrostatic field and accelerated to $\sim 40 \mathrm{eV}$ towards the entrance zone of the time of flight mass spectrometer.

A commercial reflectron time-of-flight mass spectrometer (Tofwerk AG, model HTOF) was employed to record the mass spectra. This instrument can operate in two modes: a V-mode and a W-mode. The V-mode is the standard reflectron configuration and delivers a mass resolution of $\sim 5000$ (FWHM). The orthogonal acceleration source allows a duty cycle of around $25 \%$ in the V-mode configuration. The $\mathrm{W}$-mode introduces an extra ion mirror, which essentially doubles the ion flight length. This produces a higher mass resolution but does so at the expense of a reduced detection sensitivity due to five more grids that have to be passed and lateral broadening of the ion packet, which means that more ions miss the detector. Consequently, since sensitivity was the more important factor in the current work, the mass spectrometer was operated in the V-mode. The ions are orthogonally extracted by a pulsed acceleration voltage (700 V, $4 \mu$ s pulse length) at a repetition rate of $30 \mathrm{kHz}$ and are detected by a microchannel plate detector after a flight length of about $1 \mathrm{~m}$. The base pressure in the mass spectrometer is typically $10^{-7}$ mbar. We used acquisition times from $30 \mathrm{~min}$ to $6 \mathrm{~h}$ to achieve adequate signal-to-noise ratios. After acquisition, the spectra were calibrated to a mass-accuracy of better than $0.01 \mathrm{amu}$ to account for any mass shifts, which inevitably occur over long data acquisition periods. 


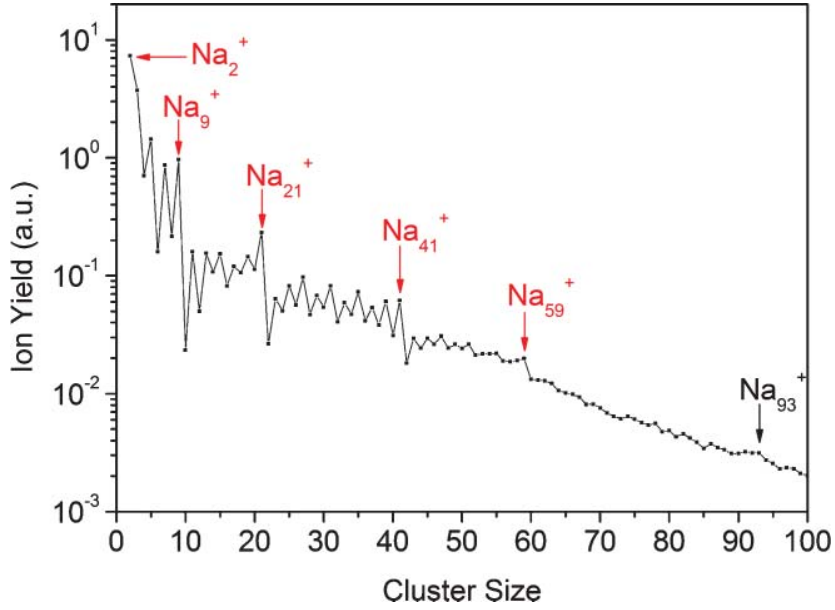

FIG. 2. Plot of the measured abundance of $\mathrm{Na}_{n}{ }^{+}$cluster ions as a function of $n$ obtained at an electron impact energy of $77 \mathrm{eV}$.

\section{RESULTS AND DISCUSSION}

\section{A. Magic numbers}

The size distribution of sodium cluster ions is illustrated by the data in Figure 2, which were obtained at an electron impact energy of $77 \mathrm{eV}$. Cluster ions with up to $\sim 100 \mathrm{Na}$ atoms were easily seen in this work. These are considerably larger than in previous studies of sodium clusters grown in/on helium nanodroplets, which have yielded maximum cluster sizes of $n=25$ for $\mathrm{Na}_{n} \cdot{ }^{6,12,13}$ However, $\mathrm{K}_{n}$ clusters with up to 70 potassium atoms have been reported. ${ }^{6,13}$ The sodium cluster size distribution at much lower electron energies is quite similar to that in Figure 2, although the absolute signal levels are markedly lower. Figure 2 shows a number of notable features, including an odd-even intensity alternation and magic numbers at $n=9,21,41,59$, and 93. Vongehr and Kresin have reported the odd-even intensity alternation for small $\mathrm{Na}_{n}{ }^{+}$clusters $(n \leq 13)$ in an earlier study of sodiumdoped helium nanodroplets. ${ }^{10}$

The odd-even intensity alternation and the observed magic numbers are well known and can be understood in terms of the electronic shell model. ${ }^{6,14}$ Cluster ions with enhanced intensities are attributed to structures with enhanced stabilities. It is worth pointing out that we would not expect to see any magic number features if the ions were efficiently cooled by the helium, since each cluster would essentially be frozen in a potential energy minimum. There would thus be insufficient thermal energy to fragment and therefore no opportunity to become trapped in a deeper potential energy well (corresponding to a magic number species). In terms of neutral $\mathrm{Na}_{n}$ clusters, Honea et al. have demonstrated essentially this point by photoionizing the clusters after cooling in helium gas, which removes most of the magic number peaks in the mass spectrum. ${ }^{15}$ The cooling of $\mathrm{Na}_{n}{ }^{+}$cluster ions by helium in the present work will be compounded by the fact that the formation of the neutral $\mathrm{Na}_{n}$ clusters will already remove much of the helium. The binding energy per atom in sodium clusters has been calculated to be approximately $0.6 \mathrm{eV} .{ }^{16}$ Assuming that each helium atom that is boiled off the droplet removes $0.6 \mathrm{meV},{ }^{11}$ then the addition of each $\mathrm{Na}$ atom as the cluster is formed should eventually release $\sim 1000$ helium atoms. In addition, each arriving $\mathrm{Na}$ atom will deliver a quantity of thermal energy that must also be dissipated and will account for the evaporation of roughly 100 additional helium atoms. Given the mean helium droplet size of $10^{5}$ helium atoms estimated in this work, then most sodium clusters with more than $90 \mathrm{Na}$ atoms are likely to have no helium atoms attached by the time ionization begins. However, for the key $\mathrm{Na}_{n}$ cluster sizes interrogated in the next section, i.e., $n<30$, the majority of $\mathrm{Na}_{n}$ clusters should still be in/on helium droplets consisting of several thousand helium atoms prior to ionization.

Given the loss of helium atoms prior to ionization and the subsequent energy release on ionization, the observation of magic number cluster ions in the current study is unsurprising. As will be discussed in more detail later, there are two options for ionization: charge transfer from $\mathrm{He}^{+}$, which essentially delivers $24.6 \mathrm{eV}$ into the sodium cluster, or Penning ionization by electronically excited helium, which is capable of delivering $19.8 \mathrm{eV}$. Given the low ionization energies of sodium clusters $(<5 \mathrm{eV})$ (Ref. 17) there will clearly be a large quantity of excess energy to dissipate, i.e., hot $\mathrm{Na}_{n}{ }^{+}$clusters will initially be formed. However, in the case of Penning ionization there is the opportunity for the departing electron to take away a considerable amount of the excess energy, so residual internal energy in the alkali cluster ion may be much lower than in the charge transfer case.

Previous studies have shown that ion cooling can be quite slow in helium nanodroplets, ${ }^{18-22}$ and so it is plausible that the hot $\mathrm{Na}_{n}{ }^{+}$clusters will initiate a fragmentation cascade which is punctuated by the added stability of certain cluster structures. This accounts for both the odd-even intensity alternations and clear magic number features reflected in Figure 2.

\section{B. Sodium cluster immersion}

The primary finding in the current work derives from the electron energy dependence of the cluster ion signals. When the sodium clusters reside within or on the surface of a reasonably large helium droplet, direct electron impact ionization of the clusters is unlikely on the basis of simple geometric probability. Consequently, the dominant ionization processes will involve initial excitation of the helium, followed by possible energy transfer to the sodium cluster. As mentioned in Sec. III A, one important threshold occurs at $19.8 \mathrm{eV}$, which corresponds to excitation of a helium atom to the metastable $2^{3} \mathrm{~S}$ state $\left(\mathrm{He}^{*}\right)$. A helium atom in this state could ionize a sodium cluster by Penning ionization if the two were to meet. Penning ionization of dopants in helium nanodroplets is well-known and has been subjected to several studies. ${ }^{23-28}$ Above $24.6 \mathrm{eV}$ ionization of atomic helium can occur to form $\mathrm{He}^{+}$. This can then transfer charge between helium atoms by a resonant charge hopping mechanism ${ }^{29-32}$ and which may eventually result in an encounter with a dopant. Since a dopant such as a sodium cluster will have a far lower ionization energy than helium, charge transfer to the cluster will terminate the charge transfer process and will be accompanied by the release of considerable energy, thus 


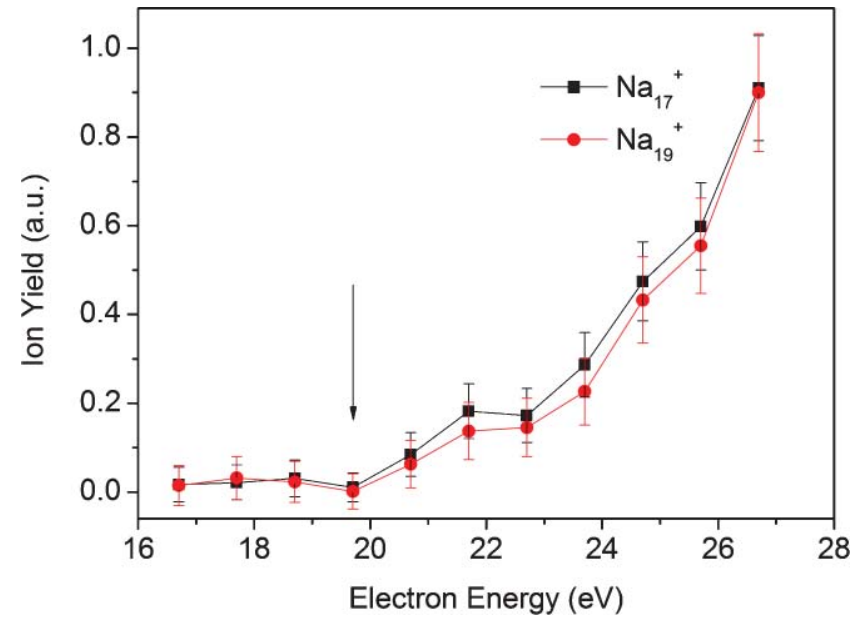

FIG. 3. Ion yield as a function of electron energy for $\mathrm{Na}_{17}{ }^{+}$and $\mathrm{Na}_{19}{ }^{+}$. The error bars were obtained from Poisson statistics, i.e., the assumption that the error is proportional to $\sqrt{ } N$ where $N$ is the number of ion counts in a particular $\mathrm{m} / \mathrm{z}$ channel. The arrow marks the point where the ion yield begins to grow above the background level.

ejecting sodium cluster ions into the gas phase. For the same reason, ion ejection is also likely if Penning ionization occurs.

As well as different threshold energies, the two ionization processes also differ in one other important aspect. For a positive hole, the potential energy is progressively lowered as it moves from the surface to the centre of the droplet. This potential energy gradient drives the positive hole along a hopping route which preferentially takes it towards the centre of the droplet. ${ }^{31,32}$ On the other hand, neutral helium in its metastable $2^{3} \mathrm{~S}$ state will favour a surface location because this avoids expending energy in creating a bubble in the interior of the liquid helium. Consequently, Penning ionization is expected to preferentially ionize dopants on the surface of a helium droplet, whereas a positive hole is more likely to attack a dopant in the interior of a droplet. These two processes should show distinct threshold energies and provide the key to distinguishing between surface and interior locations for the neutral sodium clusters.

Figure 3 shows the yields for two cluster ions, $\mathrm{Na}_{17}{ }^{+}$and $\mathrm{Na}_{19}{ }^{+}$, as a function of applied electron kinetic energy. Both show similar yield curves and they are also similar to those of smaller clusters all the way down to $\mathrm{Na}_{3}{ }^{+}$(the odd cluster ions are more convenient for investigation because they give much larger ion signals than even cluster ions at low electron energies). Ion production shows a substantial rise at around $20 \mathrm{eV}$, which is consistent with ion production by Penning ionization. It should be noted that Vongehr et al. have recorded similar data, but only for sizes up to $n=13 .{ }^{10}$

By way of contrast, Figure 4 shows yield curves for $\mathrm{Na}_{21}{ }^{+}, \mathrm{Na}_{23}{ }^{+}, \mathrm{Na}_{27}{ }^{+}$, and $\mathrm{Na}_{31}{ }^{+}$. These curves are also rather similar to each other but, crucially, they now differ significantly from the curves for the lighter clusters shown in Figure 3. Specifically, there is no detectable rise in ion signal near $20 \mathrm{eV}$, suggesting that the Penning process is unimportant in the production of these clusters. However, there is a clear rise in yield between 24 and $25 \mathrm{eV}$, which is explained by ion production by charge transfer from $\mathrm{He}^{+}$.

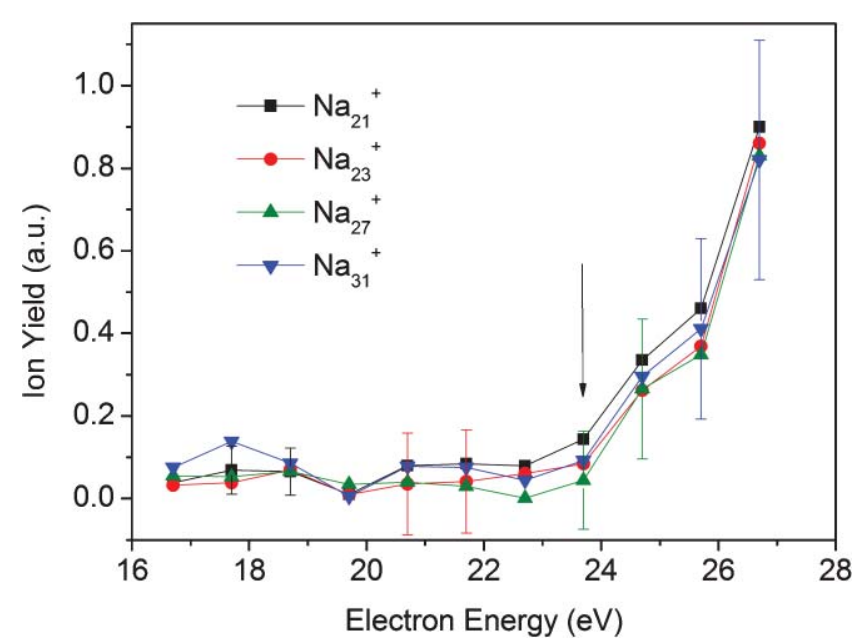

FIG. 4. Ion yield as a function of electron energy for $\mathrm{Na}_{21}{ }^{+}, \mathrm{Na}_{23}{ }^{+}, \mathrm{Na}_{27}{ }^{+}$, and $\mathrm{Na}_{31}{ }^{+}$. The arrow marks the point where the ion yield begins to grow above the background level.

The ion yield curves for $\mathrm{Na}_{19}{ }^{+}$and smaller clusters are consistent with a surface location for the corresponding neutral clusters, whereas those for $\mathrm{Na}_{21}{ }^{+}$and larger clusters are consistent with an interior location for the corresponding neutral clusters. These findings provide strong evidence for a switch from a surface location to an interior location at a certain sodium cluster size. For completeness, we show aggregated data for the much weaker even cluster ions in Figure 5, where the signal from all $\mathrm{Na}_{n}{ }^{+}$ions with $n>20$ have been summed together and similarly all of those with $n$ $\leq 20$. Again this is consistent with a change in cluster location at around the $n=20$ or 21 size. However, establishing the precise size at which this occurs is complicated by ion fragmentation, which can arise because of the considerable excess energy released regardless of whether ionization is mediated

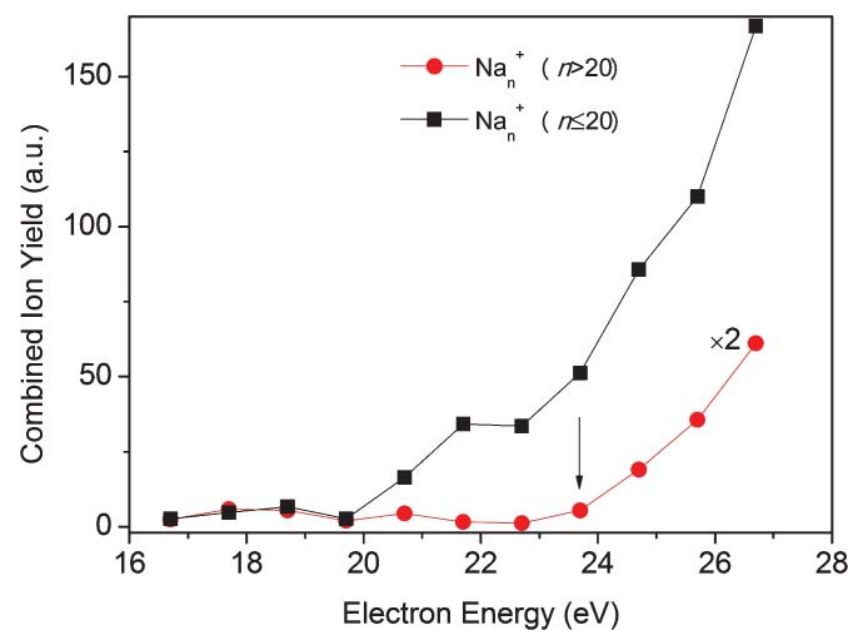

FIG. 5. Abundance distributions for the even-numbered sodium cluster ions, $\mathrm{Na}_{n}{ }^{+}$. Because the signal was weak for a given $n$, for reasons that are wellknown, the statistics are poor for individual values of $n$. Consequently, the data are presented here as two curves which result from the sum of all evennumbered ion signals in the range $10 \leq n \leq 20$ and for $n>20$, respectively. Distinct differences in the thresholds for ion yield increases are seen for the two curves. The arrow marks the point where the ion yield begins to grow above the background level. 
via $\mathrm{He}^{*}$ or $\mathrm{He}^{+}$. In the photoionization of $\mathrm{Na}_{n}$ clusters, the principal fragmentation channel involves the ejection of neutral atoms or dimers. ${ }^{33,34}$ Unfortunately, electron impact ionization has the potential to be more destructive because of the larger excess energy typically deposited into the ions and a study of small $\mathrm{Na}_{n}{ }^{+}$cluster ions $(n=1-5)$ following electron impact seems to bear this out. ${ }^{35}$ Although direct electron impact was not used to ionize the clusters in the present work, considerable excess energy is still likely to be deposited into the cluster ions. This will be most significant for charge transfer from $\mathrm{He}^{+}$, where all of the excess energy must initially appear as internal energy of the dopant ion. Thus, because of the potential for ion fragmentation, we can only firmly establish a lower limit of $n \geq 21$ for sodium cluster submersion in ${ }^{4} \mathrm{He}$ droplets. The lower limit is in excellent agreement with a recent theoretical prediction made by Stark and Kresin, whose model suggested that the minimum $\mathrm{Na}_{n}$ cluster size for submersion in ${ }^{4} \mathrm{He}$ droplets occurred at $n=21 .{ }^{9}$

\section{Formation of large $\mathrm{Na}_{n}$ clusters}

The observation of large $\mathrm{Na}_{n}{ }^{+}$cluster ions, well above the apparent size required for submersion of the neutral, raises an interesting question: how are neutral $\mathrm{Na}_{n}$ clusters above the critical size formed? As a result of ion fragmentation we cannot establish the precise size at which the neutral cluster sinks into the helium droplets. However, it is known that ejection of $\mathrm{Na}$ from $\mathrm{Na}_{n}{ }^{+}$cluster ions requires a minimum of approximately $0.6 \mathrm{eV} .^{15,33}$ If we assume a worst-case scenario and assume that all of the excess energy on ionization of the cluster is employed to evaporate $\mathrm{Na}$ atoms, then there is roughly $16 \mathrm{eV}$ to dissipate for Penning ionization and $21 \mathrm{eV}$ for charge transfer from $\mathrm{He}^{+}$. Thus, the actual surface $\rightarrow$ interior transition for the neutral $\mathrm{Na}_{n}$ cluster could occur for $n$ as large as $45-55$, if fragmentation is severe and cooling by the helium is highly inefficient. The significant point being made here is that we see ions from neutral clusters much larger than this upper limit for submersion, so the $\mathrm{Na}_{n}$ clusters must continue to grow beyond the threshold submersion size.

This poses a potential problem, since the addition of further $\mathrm{Na}$ atoms might be impeded when the main cluster becomes submerged, since any $\mathrm{Na}$ atoms added thereafter might favour a surface location. One way to explain the large cluster formation is to assume that submersion is a relatively slow process. Thus, $\mathrm{Na}$ atoms could continue to be added to the existing cluster as the cluster slowly immerses itself in the helium droplet. However, this seems unlikely given the timescale for $\mathrm{Na}$ atom addition. For a helium droplet composed of $10^{5}$ helium atoms and an assumed sodium pressure of $10^{-4}$ mbar in the pick-up cell (a likely overestimate), a simple collision frequency calculation shows that a $\mathrm{Na}$ atom will hit the helium droplet roughly every $2 \mu \mathrm{s}$, and will clearly strike the $\mathrm{Na}_{n}$ cluster even less frequently. There would seem to be no obvious mechanism for such an exceedingly slow submersion, so we suggest a more plausible alternative.

Assuming that the $\mathrm{Na}_{n}$ cluster descends into the droplet almost instantaneously once the critical size is reached, then additional $\mathrm{Na}$ atoms, which might initially adsorb at the sur- face, could be drawn into the droplet through a favourable dispersion interaction with the highly polarizable $\mathrm{Na}_{n}$ cluster. An analogous process has been observed previously in the case of $\mathrm{Ba}$ atoms, which ordinarily reside on the surface of helium droplets but which can be forced inside by first adding a highly polarizable $\mathrm{Xe}_{n}$ cluster of sufficient size. ${ }^{36}$ This mechanism is consistent with the experimental observations reported here, accounting for $\mathrm{Na}_{n}$ cluster growth well beyond the critical submersion size.

\section{CONCLUSIONS}

The submersion of sodium clusters in helium nanodroplets has been demonstrated for the first time. Using controlled electron impact energies to construct ion yield curves, a clear surface $\rightarrow$ interior transition has been shown to take place at a sufficiently large cluster size. Because of likely ion fragmentation, we can only establish a minimum size at which the cluster sinks into the helium droplet but the value obtained, $n \geq 21$, is in remarkably good agreement with a recent theoretical prediction, which suggested that $\mathrm{Na}_{n}$ sinks into a helium droplet for cluster sizes $n \geq 21$.

\section{ACKNOWLEDGMENTS}

The work was supported by the FWF, Wien (P19073) and the European Commission, Brussels. A.M.E. is grateful to the University of Innsbruck for financial support. S.D. gratefully acknowledges an APART grant from the Austrian Academy of Sciences. L.A.d.L, P.B., and C.L. thank the University of Innsbruck for support via the Doktoratsstipendium aus der Nachwuchsförderung.

${ }^{1}$ F. Stienkemeier, J. Higgens, C. Callegari, S. I. Kanorsky, W. E. Ernst, and G. Scoles, Z. Phys. D 38, 253 (1996).

${ }^{2}$ F. Ancilotto, E. Cheng, M. W. Cole, and F. Toigo, Z. Phys. B 98, 323 (1995).

${ }^{3}$ A. Nakayama and K. Yamashita, J. Chem. Phys. 114, 780 (2001).

${ }^{4}$ J. Higgins, C. Callegari, J. Reho, F. Stienkemeier, W. E. Ernst, K. K. Lehmann, M. Gutowski, and G. Scoles, J. Phys. Chem. A 102, 4952 (1998).

${ }^{5}$ J. H. Reho, J. Higgins, M. Nooijen, K. K. Lehmann, G. Scoles, and M. Gutowski, J. Chem. Phys. 115, 10265 (2001).

${ }^{6}$ J. Tiggesbäumker and F. Stienkemeier, Phys. Chem. Chem. Phys. 9, 4748 (2007)

${ }^{7}$ S. Vongehr and V. V. Kresin, J. Chem. Phys. 119, 11124 (2003).

${ }^{8}$ O. Bünermann and F. Stienkemeier, Eur. Phys. J. D 61, 645 (2011).

${ }^{9}$ C. Stark and V. V. Kresin, Phys. Rev. B 81, 085401 (2010).

${ }^{10}$ S. Vongehr, A. A. Scheiemann, C. Wittig, and V. V. Kresin, Chem. Phys. Lett. 353, 89 (2002).

${ }^{11}$ J. P. Toennies and A. F. Vilesov, Angew. Chem. Int. Ed. 43, 2622 (2004).

${ }^{12}$ C. P. Schulz, P. Claas, D. Schumacher, and F. Stienkemeier, Phys. Rev. Lett. 92, 013401 (2004)

${ }^{13}$ G. Droppelmann, M. Mudrich, C. P. Schulz, and F. Stienkemeier, Eur. Phys. J. D 52, 67 (2009).

${ }^{14}$ D. M. P. Mingos and Z. Lin, Chem. Phys. 137, 15 (1989).

${ }^{15}$ E. C. Honea, M. L. Homer, J. L. Persson, and R. L. Whetten, Chem. Phys. Lett. 171, 147 (1990).

${ }^{16}$ I. A. Solov'yov, A. V. Solov'yov, and W. Greiner, Phys. Rev. A 65, 053203 (2002).

${ }^{17}$ H. Gölich, T. Lange, T. Bergmannm, U. Näher, and T. P. Martin, Chem. Phys. Lett. 187, 67 (1991).

${ }^{18}$ S. Yang, S. M. Brereton, M. D. Wheeler, and A. M. Ellis, J. Phys. Chem. A 110, 1791 (2006). 
${ }^{19}$ S. Yang, S. M. Brereton, M. D. Wheeler, and A. M. Ellis, Phys. Chem. Chem. Phys. 7, 4082 (2005).

${ }^{20}$ S. Yang, S. M. Brereton, and A. M. Ellis, Intl. J. Mass. Spectrom. 253, 79 (2006).

${ }^{21}$ S. Denifl, F. Zappa, I. Mähr, A. Mauracher, M. Probst, T. D. Märk, and P. Scheier, J. Am. Chem. Soc. 130, 5065 (2008).

${ }^{22}$ H. Schöbel, M. Dampc, F. F. da Silva, A. Mauracher, F. Zappa, S. Denifl, T. D. Märk, and P. Scheier, Int. J. Mass Spectrom. 280, 26 (2009).

${ }^{23}$ M. Lewerenz, B. Schilling, and J. P. Toennies, J. Chem. Phys. 102, 8191 (1995).

${ }^{24}$ A. A. Scheidemann, V. V. Kresin, and H. Hess, J. Chem. Phys. 107, 2839 (1997).

${ }^{25}$ S. Denifl, M. Stano, A. Stamatovic, P. Scheier, and T. D. Märk, J. Chem. Phys. 124, 054320 (2006).

${ }^{26}$ U. Henne and J. P. Toennies, J. Chem. Phys. 108, 9327 (1998).

${ }^{27}$ F. Zappa, S. Denifl, I. M Mähr, A. Bacher, O. Echt, T. D. Märk, and P. Scheier, J. Am. Chem. Soc. 130, 5573 (2008).
${ }^{28}$ H. Schöbel, P. Bartl, C. Leidlmair, M. Daxner, S. Zöttl, S. Denifl, T. D. Märk, P. Scheier, D. Spånberg, A. Mauracher, and D. K. Bohme, Phys. Rev. Lett. 105, 243402 (2010).

${ }^{29}$ A. Scheidemann, B. Schilling, and J. P. Toennies, J. Phys. Chem. 97, 2128 (1993).

${ }^{30}$ B. E. Callicoatt, K. Förde, T. Ruchti, L. Jung, and K. C. Janda, J. Chem. Phys. 108, 9371 (1998).

${ }^{31}$ W. K. Lewis, M. Lindsay, R. J. Bemish, and R. E. Miller, J. Am. Chem. Soc. 127, 7235 (2005).

${ }^{32}$ A. M. Ellis and S. Yang, Phys. Rev. A 76, 032714 (2007).

${ }^{33}$ C. Bréchignac, P. Cahuzac, J. Leygnier, and J. Weiner, J. Chem. Phys. 90, 1492 (1989)

${ }^{34}$ I. V. Hertel, C. P. Schulz, A. Goerke, H. Palm, and G. Leipelt, J. Chem. Phys. 107, 3528 (1997).

${ }^{35}$ L. Bewig, U. Buck, C. Mehlmann, and M. Winter, J. Chem. Phys. 100, 2765 (1994)

${ }^{36}$ E. Lugovoj, J. P. Toennies, and A. Vilesov, J. Chem. Phys. 112, 8217 (2000). 